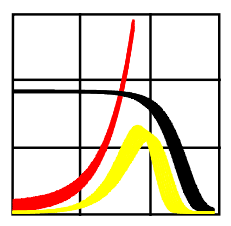

Max-Planck-Institut für demografische Forschung

Max Planck Institute for Demographic Research

Doberaner Strasse 114 - D-18057 Rostock · GERMANY

Tel +49 (0) 3812081 - 0; Fax +49 (0) 3812081 - 202;

http://www.demogr.mpg.de

MPIDR WORKING PAPER WP 1999-007

JULY 1999

The Swedish Baby Bomb and Bust of 1985-1996

Revisited: the Role of Tempo, Quantum and Variance Effects

Hans-Peter Kohler (kohler@ demogr.mpg.de)

(C) Copyright is held by the author.

Working papers of the Max Planck Institute for Demographic Research receive only limited review. Views or opinions expressed in working papers are attributable to the author and do not necessarily reflect those of the Institute. 


\title{
The Swedish Baby Boom and Bust of 1985-1996 Revisited: the Role of Tempo, Quantum and Variance Effects
}

\author{
Hans-Peter Kohler*
}

July 15, 1999

\begin{abstract}
Variance effects, i.e., increases in the standard deviation of the fertility schedule over time, constitute a systematic and interesting aspect of recent fertility patterns in Europe. In this paper we investigate the relevance of these variance changes for the evaluation of the Swedish baby boom and bust in terms of tempo and quantum effects. Using an extension of the adjusted $T F R$ that incorporates variance changes, we show that there was no reduction in the pace of postponement of first births in Sweden during the period 1980-1995. Thus, the baby boom was overwhelmingly due to quantum effects, and did not have a relevant tempo component as suggested by the TFR-adjustment without variance effects. Moreover, the tempo changes during this period clearly reflect the implications of the policy changes in the 1980s: while the tempo for first births remained relatively constant, the tempo at higher orders was reduced substantially after the extension of policies that favor short interbirth intervals. However, this pattern is not visible in the tempo effects without adjustment for variance effects. We therefore argue that variance effects deserve further attention in the investigation of recent fertility patterns and in the adjustment of the total fertility rate.
\end{abstract}

\section{Introduction}

Swedish fertility during the 1980s and 1990s has been of great interest to demographers for its distinct and unusual pattern. Whereas fertility levels stagnated or declined in many European countries during the 1980s, Sweden experienced a baby boom after 1985. Between 1985 and 1990 the TFR increased from 1.68 to a level of 2.14, exceeding replacement level. Surprisingly, this upsurge in fertility was accompanied by an increasing participation of women in the labor force: between 1985

${ }^{*}$ Head of Research Group on Social Dynamics and Fertility, Max Planck Institute for Demographic Research, Doberaner Str. 114, 18057 Rostock, Germany. Email: kohler@demogr.mpg.de, www: http://user.demogr.mpg.de/kohler. 
and 1990 female labor force participation rose from an already high level of $78 \%$ to $81 \%$, the highest level in Europe at the time (OECD 1998). The increase in Swedish fertility during the late 1980s has been attributed to generous and expanding social policies favoring gender equality and female labor force participation, to decreasing opportunity costs of fertility, a flourishing Swedish economy with low unemployment rates, and to optimistic evaluations of economic conditions and opportunities for the future (Andersson 1999; Gustafsson et al. 1996; Hoem 1990; Hoem and Hoem 1997a, 1997b; Meisaari-Polsa and Söderström 1995; Sundstrom and Stafford 1992; Walker 1995). In the 1990s this baby boom was displaced by an equally swift baby bust, and by 1997 the total fertility rate had declined to a historically low level of 1.53 (Council of Europe 1998). The 1990s were associated with a worsening of economic conditions and a subsequent reduction in social support for families. These factors are thought by many to underlie the reversal of the fertility trend at the beginning of the 1990s (Hoem 1998; Hoem and Hoem 1997b).

Sweden has often been perceived as being a demographic forerunner with high levels of cohabitation, out-of-wedlock childbearing, and a substantial postponement of fertility to higher ages. For instance, at least since the early 1970s the mean age at first birth has been rising steadily. During the late 1980s, however, this postponement of fertility lost some of its pace: the baby boom was in part due to an increase in the fertility rates at younger ages, especially below age 25 (Hoem and Hoem 1997a). While the mean age at first birth rose between 1975 and 1985 from 24.5 to 26.1 years, it only increased marginally to 26.3 years during the baby boom period up to 1990. A second component of the baby boom was a reduced interbirth interval. This effect was especially noticeable for second and third births, and it is usually attributed to incentive changes in the public benefit programs introduced in 1980 and 1986 (Hoem 1990). After 1990, the postponement of births regained its pace as fertility declined, and by 1995 the mean age at first birth had risen to 27.3 years (Council of Europe 1998).

The Swedish baby boom and bust is therefore associated with substantial changes in the tempo of fertility, i.e., in the extent to which fertility is postponed towards higher ages over time. This varying tempo of fertility makes Sweden a particularly relevant case for the application of the adjusted total fertility rate (Bongaarts and Feeney 1998). The adjusted TFR is equivalent to the total fertility rate that would have been observed if there had been no change in the tempo of fertility during a calendar year. On the one hand, fluctuations in the observed $T F R$ that are not mirrored in the adjusted $T F R$ are merely due to variations in the tempo of fertility; these fluctuations would have been absent if the tempo changes had been absent during the respective period. On the other hand, fluctuations in the adjusted $T F R$ represent changes in the fertility level that are not due to changes in the postponement of births. They are therefore quantum effects which reflect variations in the level of fertility after the effects of tempo changes have been removed.

Since Sweden experienced substantial changes in the tempo of fertility between 1985 and 1996, 
it is crucial to determine to what extent the increase and subsequent decrease in fertility after 1985 was due to tempo or quantum effects. Moreover, is the type of fertility increase in the late 1980s in Sweden typical for situations when the postponement of births slows down, and can a similar increase be expected, for instance, in Italy or Spain, if the tempo of fertility should slow down in these countries?

In seeking the answers to these questions, we shall pay particular attention to the question of whether the inferences obtained from the $T F R$ adjustment are possibly distorted due to deviations from the underlying assumptions. In particular, we focus on the assumption of no cohort effects (or equivalently, no age-period interactions). The TFR adjustment in Bongaarts and Feeney (1998) assumes that fertility may be influenced by period, age, parity and duration since last birth, but not by cohort. This absence of age-period interactions thus implies that the shape of the fertility schedule remains constant over time. Kohler and Philipov (1999) have recently investigated one particular deviation from this assumption. They have shown that an increasing variance in the (order-specific) fertility schedule leads to a bias in the observed mean age at birth and also in the adjusted total fertility rate.

In this paper we argue that the Swedish fertility rate was subject to substantial variance effects, i.e., changes in the standard deviation of the fertility schedule, after 1985. In a period with high female labor force participation, the increased incentives for children in the mid-1980s led to a diversification of fertility behavior. This diversification manifested itself in a widening fertility schedule, primarily but not exclusively, for first births. The later reduction in female labor force participation due to the worsening economic situation and a generally lower fertility level after 1990 reduced the extent of these variance effects. However, the overall trend towards a widening of the fertility schedule at each birth order remains also in the 1990s.

Including these variance effects in the analysis controls for the major changes in the shape of the Swedish fertility schedule in the 1980s and 1990s. More importantly, the incorporation of these variance effects reverses some of the conclusions obtained from the adjustment of the total fertility rate: while the adjusted $T F R$, according to Bongaarts and Feeney (1998), suggests that a significant part of the fertility increase in the late 1980s was due to a reduced tempo effect for first births, the adjusted $T F R$ with variance effects suggests for first births that the tempo of fertility remained almost constant throughout the period of the baby boom and bust. After accounting for the changing variance in the fertility schedule, first births continued to be postponed by about 0.13-0.14 years annually without any major changes in the tempo of fertility from 1985 to 1995 .

In addition, once variance effects are included, the annual changes in the order-specific mean age at birth reflect clearly the impact of the policy changes during the 1980s: while the postponement of first births continued throughout the period 1985-1995 at an almost constant pace, the postponement of second and third births was substantially reduced after 1980 and again after 1986. This pattern 
is consistent with the argument that a changing family policy in the 1980s led to a reduction in the interbirth intervals due to a 'speed premium' (Hoem and Hoem 1997a), i.e., a benefit scheme that provides an incentive for spacing the births of children closely. This differential development of tempo effects by birth-order as a result of family policy is almost undetectable if one doesn't account for the changing variance in the fertility schedule in the calculation of the mean age.

In the next section we describe the Swedish fertility pattern as of 1975 and apply the adjusted total fertility rate to the baby boom and bust period. In Section 3 we investigate in detail the fertility schedule for first births and show that variance effects account for the major changes in the fertility schedule in the 1980s and 1990s. We also provide an intuitive explanation for the incorporation of variance effects into the adjustment of the total fertility rate and then juxtapose the results from the TFR adjustment with variance effects with those of the simpler Bongaarts-Feeney adjustment (i.e., without variance effects). In Section 4 we relate the occurrence of variance effects to the major changes in public policy and labor market conditions after 1985. Section 5 discusses the relevance of variance effects for other Western and Eastern European countries and concludes the paper.

\section{The Swedish fertility pattern since 1980}

Figure 1 depicts the total fertility rate for the period 1975-1996, the Bongaarts-Feeney (BF) adjusted total fertility rate and the mean age at birth for first births (left graph) and all births (right graph). The figure reemphasizes the fertility dynamics already discussed above. The fertility decline associated with the second demographic transition (van de Kaa 1987) reached a plateau around 1977, and the TFR remained relatively constant until 1984. After 1984 a baby boom occurred and fertility increased rapidly towards a peak in 1990. About $50 \%$ of this increase is due to a rise in the $T F R$ for first births. Somewhat smaller increases occurred for second and third births. After 1990 the fertility trend reversed, and the baby boom was followed by an equally swift baby bust. In 1996 the TFR for first and all births reached its lowest level of $1.61{ }^{1}$

The increase in fertility was associated with a retardation in the postponement of births, and the mean age at first births started to level off in 1985 after a long trend towards later childbearing. Although this effect was most pronounced for first births, the tempo also slowed down for births of higher order. After 1990, the postponement of births regained its earlier pace, and the mean age at birth increased rapidly as the fertility level declined.

In the initial phase of the fertility increase, the adjusted $T F R$ is relatively constant, and the observed TFR converges towards its adjusted counterpart (Figure 1). For first births the difference between the observed and adjusted TFR decreases from 0.16 in 1983 to 0.03 in 1988, and for all births the difference decreases from 0.27 to 0.06 during the same period. The comparison with the adjusted $T F R$ therefore suggests that the increase in fertility during this period is to a significant extent due to a decline in the tempo of fertility: most of the increase in the TFR until 1988 is 
TFR and adjusted TFR (BF), first births

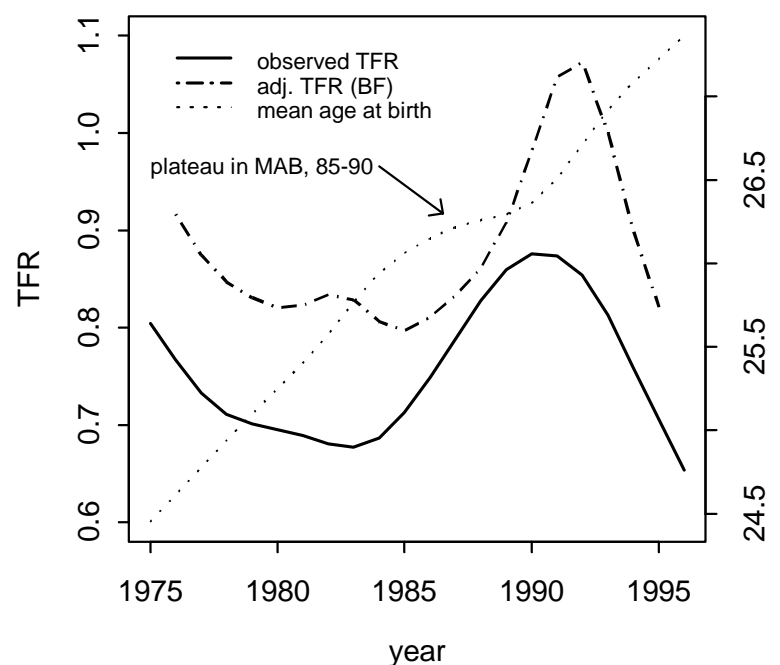

TFR and adjusted TFR (BF), all births

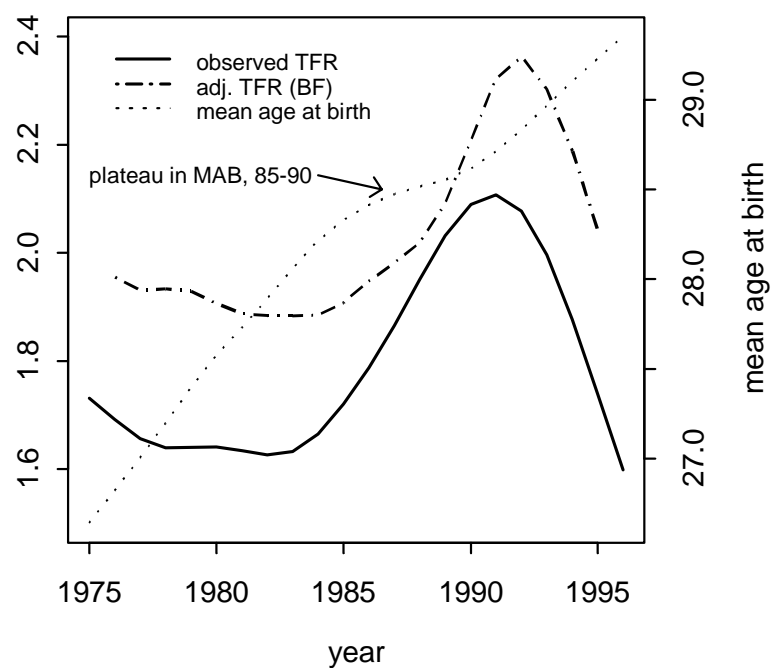

Figure 1: Total fertility rate, Bongaarts-Feeny $(\mathrm{BF})$ adjusted $T F R$, and mean age at birth for first births (left graph) and all births (right graph) in Sweden, 1975-96

explained by the reduced pace of postponement.

After 1988 the situation changes. The adjusted and observed TFR diverge considerably as the tempo of fertility starts to increase in the late phase of the baby boom. The adjusted $T F R$ for first as well as for all births peaks in 1992, two years after the observed $T F R$ had attained it highest level. Thus, the initial decline in the $T F R$ after 1990 seems to be due to the reoccurrence of a strong tempo effect. ${ }^{2}$ However, after 1992 the adjusted $T F R$ declines considerably, suggesting a substantial quantum component in the recent drop in the fertility rate.

The comparison of the observed and the adjusted $T F R$ therefore yields a differentiated picture of the baby boom and bust in terms of the relevance of tempo versus quantum effects. Initially, the reduced pace of the postponement of births leads to an increase in the observed $T F R$ at a relatively constant level of the adjusted $T F R$. Such a pattern is to be expected in Italy, Spain or Eastern Europe, where the adjusted $T F R$ considerably exceeds the observed $T F R$ if the postponement of fertility should reduce its pace. As tempo effects diminish, the observed $T F R$ converges towards the adjusted total fertility rate since the latter reflects the $T F R$ that would have been observed in the absence of the tempo effect.

After 1988, quantum components seem to dominate the development of the TFR: adjusted total fertility increases rapidly and reaches a maximum in 1992. Thereafter it drops considerably to a level of 0.82 (first births) and 2.04 (all births). Hence, the decline after 1992 cannot be attributed 
to a mere delay in childbearing. Rather, it is due to a drop in the quantum of fertility.

Before accepting the above conclusions, it is important to determine whether the inferences are warranted in terms of the underlying assumptions. In particular, is the assumption that there are no age-period interactions confirmed by the empirical pattern? If not, are the deviations from the underlying assumptions substantively relevant for the $T F R$-adjustment?

In the next section, we investigate the fertility patterns for first births in Sweden and we argue that there are systematic changes in the shape of the fertility schedule. These changes can, however, be incorporated to a substantial extent into the adjustment of the $T F R$ by accounting for variance effects. The next section shows to what extent the above-mentioned inferences change because the fertility developments in Sweden deviate from the assumptions of the TFR-adjustment.

\section{Tempo versus quantum: do variance effects matter?}

The top left graph in Figure 2 depicts the age-specific fertility rate for first births in Sweden from 1984 to 1996. The sequence of these fertility schedules reflects on one hand the increase in fertility rates - particularly at relatively young ages - from 1984 to 1990. On the other hand, the schedules after 1990 illustrate both the decline and the renewed postponement of fertility during the baby bust.

The top right graph in Figure 2 reports the fertility schedules after they have been standardized so as to exhibit the same fertility level and the same mean age at birth. Thus, in moving from the top left to the top right graph in Figure 2 we have standardized for all aspects of the fertility change that are consistent with the assumptions of the BF-adjustment of the TFR. This means that any remaining differences between the fertility schedules in the top right graph constitute deviations from the assumptions underlying the adjusted total fertility rate.

The most noticeable difference between the fertility schedules depicted in the top left graph is the trend toward an increased width, or an increased variance, of the fertility schedule. While the schedule for 1984 is most highly concentrated around its peak at age 25, the schedule for 1996 is markedly wider. Thus, during the period 1984-1996 fertility became more spread out and diversified across ages.

The bottom left graph in Figure 2 shows the standard deviation of the fertility schedule from 1974 to 1996. Until about 1985 this deviation was relatively constant with some minor fluctuations around the level of 4.55. With the onset of the baby boom in 1985, the variance started to increase significantly - a tendency that has not stopped ever since.

The bottom right graph shows the fertility schedules after they have been additionally standardized for the changing variance. The schedules in this graph exhibit not only the same fertility level and mean age at birth, but also the same standard deviation.

Most of the differences between the fertility schedules vanish after they have been additionally 

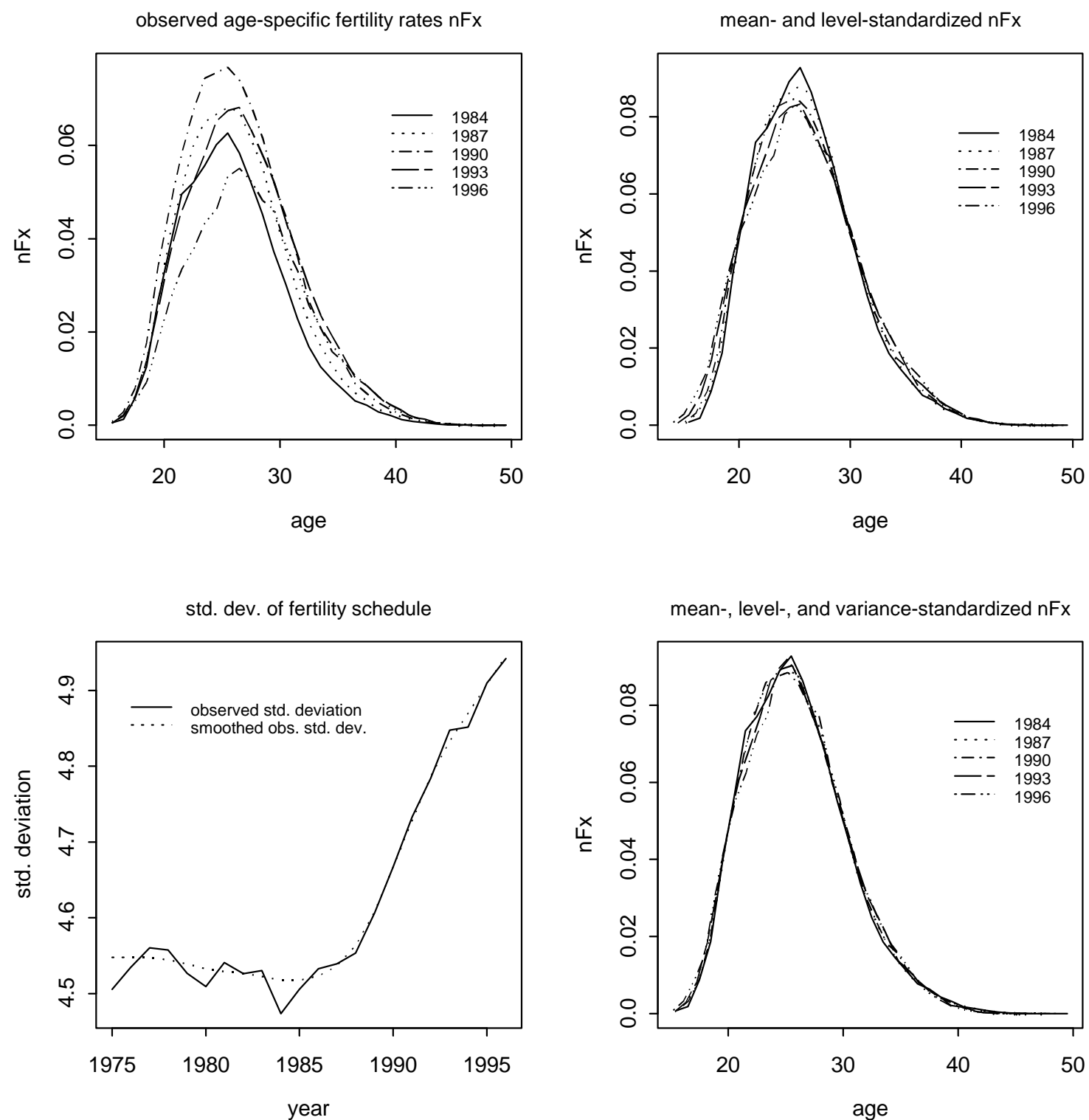

Figure 2: Fertility schedules for first births in Sweden. The top left-hand graph shows the observed age-specific fertility rates, and the top right-hand graph shows the fertility schedules standardized to exhibit the same mean age at birth and the same level of fertility. The bottom left-hand graph shows the development of the standard deviation in the fertility schedule from 1975 to 1996 . The bottom right-hand graph depicts the fertility schedules after standardization to the same fertility level, the same mean age at birth, and the same standard deviation 
standardized for the variance. Thus, incorporating variance changes is a major step towards including changes in the fertility schedule that extend beyond the two aspects accounted for by the BongaartsFeeney adjustment, namely, changes in the fertility level and shifts in the mean age. The three influences tempo, quantum and variance effects therefore provide a good characterization of fertility changes even during periods of quite substantial transformations in the fertility pattern. (See Kohler and Philipov 1999 for the application of variance effects to Hungary and Italy, where a similarly close correspondence between fertility schedules over time is obtained after - and only after - the fertility schedules are standardized for quantum, tempo and variance effects.)

How relevant are these increases in the variance of the fertility schedule for the adjustment of the $T F R$ ? For instance, are the conclusions obtained in Section 2 robust against these variance effects, or does the incorporation of these effects necessarily lead to a different interpretation of the Swedish baby boom and bust?

Kohler and Philipov (1999) have formally investigated the sensitivity of the Bongaarts-Feeney $T F R$ adjustment with respect to changes in the variance of the fertility schedule. They have shown that during periods with a rising mean age at birth, an increasing variance in the fertility schedule leads to a downward bias in $(a)$ the mean age at birth, $(b)$ the estimated tempo effect, and $(c)$ the adjusted $T F R$. These biases are particularly strong when the variance effects increase over time, as for instance during the period 1985-1990 in Sweden (see the bottom left graph in Figure 2).

The specific formal derivations in Kohler and Philipov (1999) are beyond the scope of the present paper, but the idea is straightforward and can be easily expressed. The crucial insight underlying the adjustment of the total fertility rate in Bongaarts and Feeney (1998) is that the presence of a tempo effect reduces the observed fertility levels. While in Bongaarts and Feeney (1998) these tempo effects are identical for all ages, the tempo effects in Kohler and Philipov (1999) can depend on age in a systematic manner. In particular, if the tempo effects are relatively smaller below and relatively larger above the mean age at birth, the standard deviation in the fertility schedule increases over time. This increase in the standard deviation is called the variance effect. A measure for the extent of these variance effects is the relative increase in the standard deviation of the fertility schedule per year, similar to the annual change in the mean age at birth that reflects the extent of tempo effects.

Since the tempo effect in Kohler and Philipov (1999) can depend on age, fertility rates at different ages can be affected differentially by the postponement of births. When the variance increases, which means that the tempo change is relatively lower for ages below the mean age, these age-specific fertility rates are reduced less by the postponement of births than are age-specific fertility rates above the mean age. Thus, fertility rates below the mean age differ less from the fertility rates that would have been observed in the absence of any tempo and variance changes than do fertility rates above the mean age at birth. This differential effect of postponement implies that the observed mean age at birth is biased downward in periods with an increasing mean age and standard deviation. 
adj. TFR with variance effects, first births

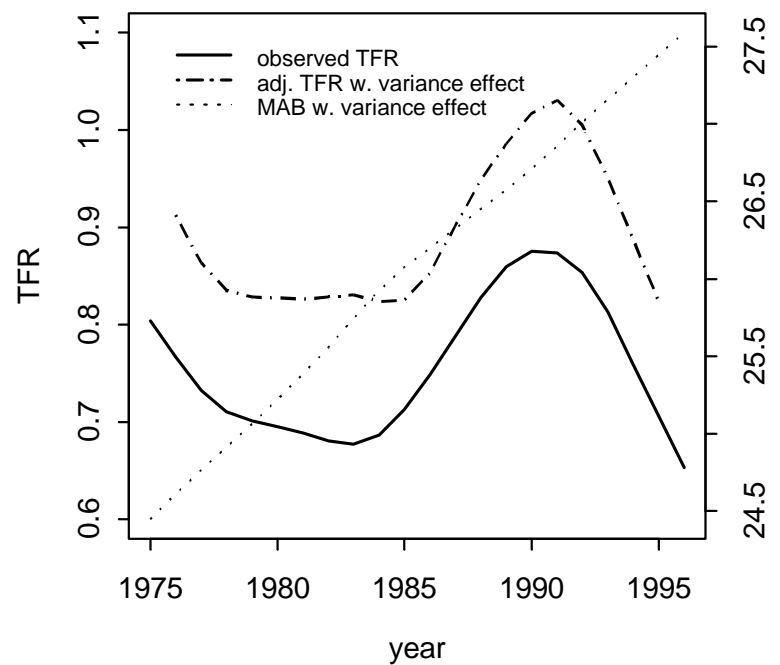

adj. TFR with variance effects, all births

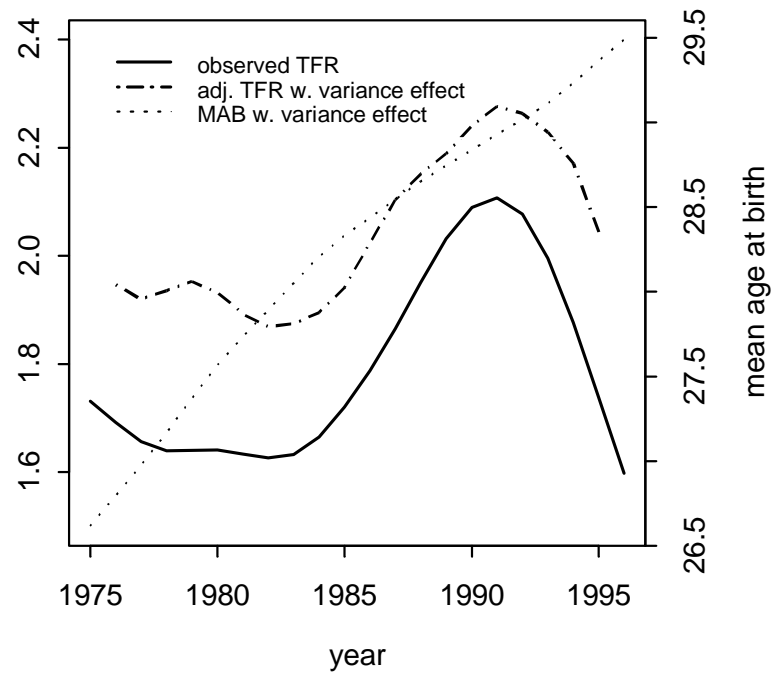

Figure 3: Total fertility rate, adjusted $T F R$ with variance effects, and mean age at birth (adjusted for variance effects) for first births (left) and all births (right) in Sweden, 1975-1996

This distortion occurs because the age-specific tempo changes lead to larger reductions in fertility rates at higher ages than at lower ages. This bias in the mean age leads to an underestimation of tempo effects, which are calculated by taking differences between the mean age at birth in different years. This effect subsequently leads to a downward bias in the adjusted total fertility rate.

Figure 3 displays the adjusted total fertility rate with variance effects for first births and all births in Sweden from 1975 to 1996. The figure exhibits some striking differences from Figure 1, which depicted the BF-adjusted $T F R$ without variance effects. ${ }^{3}$

Consider first the mean age at first birth in the graph on the left (dotted line). While the BF-adjustment in Figure 1 exhibited a plateau for the mean age at birth during the baby-boom phase 1985-1990, this plateau has completely vanished here. The postponement of births, after accounting for variance effects, does not noticeably reduce its pace throughout the period 19751996. It continues at an approximately constant rate throughout the baby boom and bust.

How is this reversal of our earlier conclusion about the tempo changes during the period 19851996 possible? The answer to this question has to do with the implications of variance effects. At the same time when fertility starts to increase around 1985, the fertility schedule starts to shift towards a wider dispersion and increased variance. In Section 4 we will argue that these two changes in the level of fertility and the variance of the fertility schedule are inherently connected to each other and to the socioeconomic situation in Sweden at the time. For the moment, however, we focus on 
the demographic implications of this development. As argued above, the onset of increases in the variance of the fertility schedule leads to a downward bias in the observed mean age. It turns out that the plateau in the mean age at first birth, which is depicted by the dotted lines in Figure 1, is due precisely to this bias. Once the bias caused by the variance changes is removed, the plateau vanishes as well.

Hence, the onset of the fertility increase in 1985 was not associated with a reduction in the tempo of fertility. It was, however, associated with the onset of variance changes that led to a larger dispersion of fertility across ages. These variance changes led to a bias in the observed mean age, and this bias after 1985 manifested itself as a plateau in the development of the mean age. However, this plateau is not due to a reduced pace of postponement. It is merely a consequence of the distortions caused by variance effects.

This different interpretation of the role of tempo effects during the Swedish baby boom and bust has direct consequences for the adjusted total fertility rate. Consider first births in the left graph of Figure 3: while in the analysis without variance effects in Figure 1 we observe a convergence between the observed and adjusted TFR during the period 1984-1988, which suggests that the fertility increase was caused by reduced tempo effects, the adjusted and observed total fertility rate now rise and fall in almost perfect accord. Thus, the incorporation of variance effects suggests that the baby boom and bust for first births from 1985 to 1996 does not have a strong tempo component. The increase and decrease in fertility during this period are due almost entirely to quantum effects.

The right graph in Figure 3 reports the respective results for all births, and it leads to similar conclusions. The convergence between the observed and adjusted $T F R$ in Figure 1 without variance effects vanishes after the changes in the shape of the fertility schedule are included in the analysis. In the right graph of Figure 3, the adjusted $T F R$ with variance effects and the observed $T F R$ increase and decrease almost synchronously, suggesting that the baby boom and bust are almost exclusively due to quantum effects. Our earlier finding, which suggested a relevant tempo effect in the initial increase of fertility around 1985, vanishes almost completely after variance effects are included in the adjustment of the $T F R$.

The left graph in Figure 4 shows the trend in the standard deviations of the fertility schedules for first, second and third births. Clearly, the relative magnitude of variance effects, i.e., changes in the standard deviation of the fertility schedule over time, is largest for first births, as is the pace of these changes. Second births exhibit a modest increase in the standard deviation of the fertility schedule during the period, and third births initially exhibit a declining variance and after 1987 an increasing variance.

The right graph in Figure 4 reports the observed mean age for first, second and third births from 1975 to 1985. Also included are the respective mean ages after removing the distortions caused by variance effects. A comparison of the mean ages with and without correction for variance effects 
std. dev. of fertility schedule

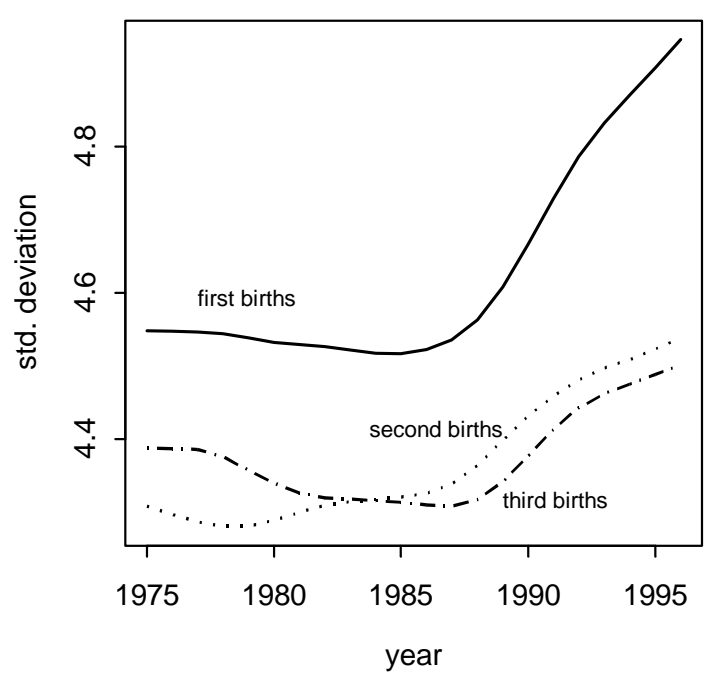

mean age at birth

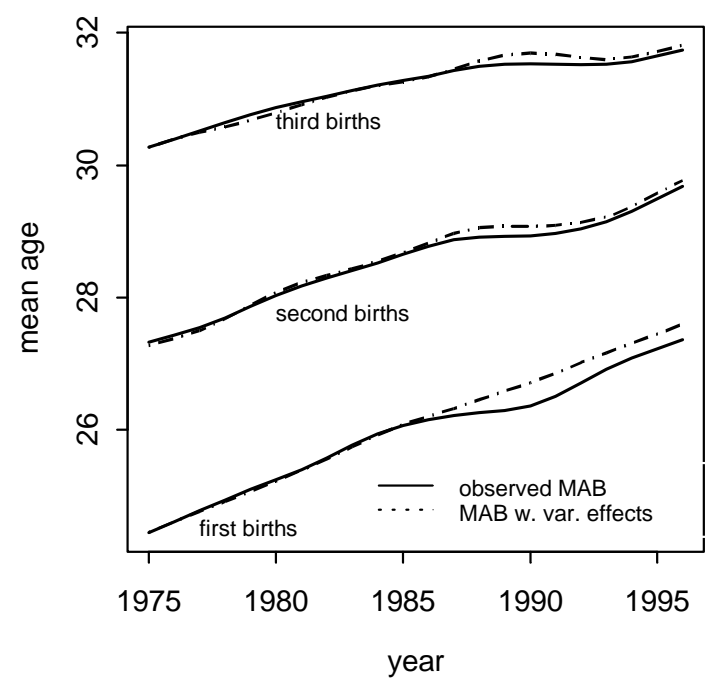

Figure 4: Standard deviation of the fertility schedule and mean age at birth for first, second and third births in Sweden, 1975-1996

reveals that distortions in the mean age, which are caused by changes in the shape of the fertility schedule, are most relevant for first births. For first births, we see in this graph once again that the plateau in the mean age after 1985 vanishes after controlling for variance changes. For second and third births a plateau after 1985 remains, but these plateaus occur slightly delayed when variance effects are accounted for.

To summarize the findings of this section, we conclude that changes in the fertility schedule constitute potentially relevant aspects in the adjustment of the total fertility rate, and that the incorporation of variance effects can lead to substantially different inferences regarding the relative importance of tempo versus quantum components of fertility change. In the Swedish case, the variance of the fertility schedule started to increase along with the level of fertility in 1985 . The incorporation of this effect removes the plateau in the observed mean age at first birth, and thus the conclusion that a reduced tempo of fertility led to the initial increase in fertility no longer holds. Especially for first births the TFR adjustment with variance effects suggests that the Swedish baby boom and bust are due to quantum and variance effects, but not to changes in the tempo of fertility. This finding is in sharp contrast to the inferences obtained from the application of the Bongaarts and Feeney (1998) adjustment. Although the variance effects are strongest for first births, similar patterns of changes in the fertility schedule are also to be observed for second and third births. Thus, the finding that changes in the tempo of fertility do not seem to be a major factor in the baby 
boom and bust also holds when births of all orders are considered.

This finding also implies that the Swedish baby boom during the late 1980s does not provide a good example for the type of fertility increases that are to be expected once the postponement of births decelerates in Italy, Spain or other countries with presently very low fertility and large tempo effects. Once variance effects are included in the analysis, no substantial reduction in the tempo is found for first births during the Swedish baby boom. Thus, the Swedish experience during the late 1980s does not provide evidence that substantial reductions in the postponement of first births are likely to occur in developed countries. Based on these analyses we therefore argue that it remains an open question whether reductions in the tempo of fertility are likely to occur in Italy, Spain or Eastern European countries in the near future.

\section{Socioeconomic changes and the Swedish fertility pattern}

Given the importance of variance effects for understanding the recent fertility change in Sweden, it is necessary to investigate whether these variance effects are systematically related to changes in the socioeconomic context and in family policies in Sweden.

The top graphs in Figure 5 show the developments in the female labor force participation (horizontal axis) and the total fertility rate for first and third births (vertical axis) from 1975 to 1996. Until 1984 an increasing female labor force participation $(L F P)$ rate is associated with declining fertility for first births. After 1984 the rather unusual combination of a further increase in the female $L F P$ rate and the $T F R_{1}$ is to be observed, which comes to a peak in 1990 for both female labor force participation and the fertility rate for first births (note: the $T F R$ with subscript $i$ refers to the total fertility rate of order $i$; i.e., $T F R_{1}$ denotes the total fertility rate for first births). After 1990 both female $L F P$ and the $T F R_{1}$ retract again, and in 1995 they return almost to levels that had prevailed a decade earlier.

For third births the period 1975-1982 was characterized by an increase in the female LFP rate and a modest increase in the $T F R_{3}$ (see the top right graph in Figure 5). After 1984 a swift rise in the fertility for third births occurs with the onset of the baby boom. At the same time, female labor force participation continues to increase. After 1990-1991 the $T F R_{3}$ declines, together with a decrease in the female $L F P$ rate and a worsening of the general economic conditions.

Hoem (1990) and Hoem and Hoem (1997b) argue that the increase in the fertility at all orders during the 1980s is due to favorable labor market conditions, a widespread optimism regarding future social and economic conditions and massive investment in social policies directed towards families. Most relevant for our context here are the differential incentives caused by the policy changes in the 1980s for the timing of births of different orders. In 1980 and 1986 the Swedish government extended policies that favored a rapid succession of subsequent births after the first or second child. In particular, if the next child was born within 24 (up to 1985) or 30 (after 1986) 
First Births
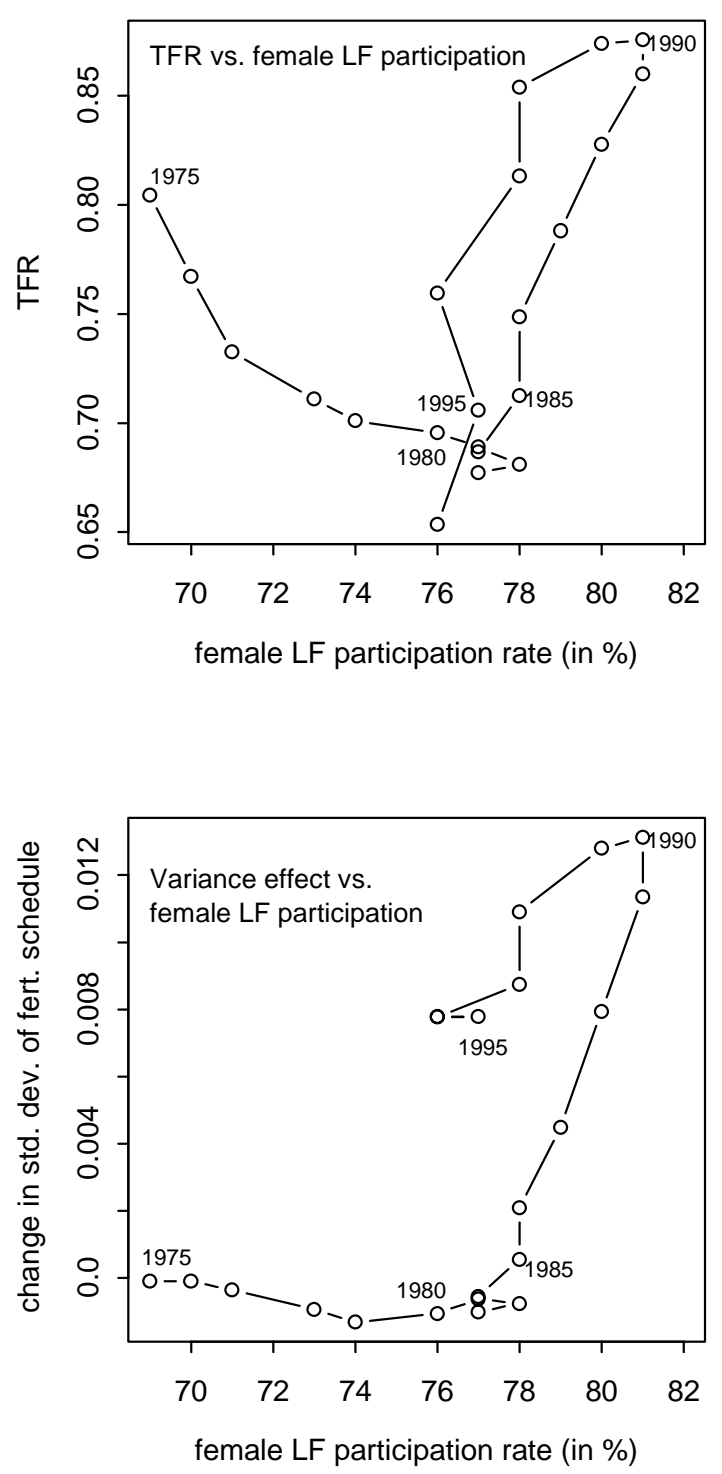

\section{Third Births}
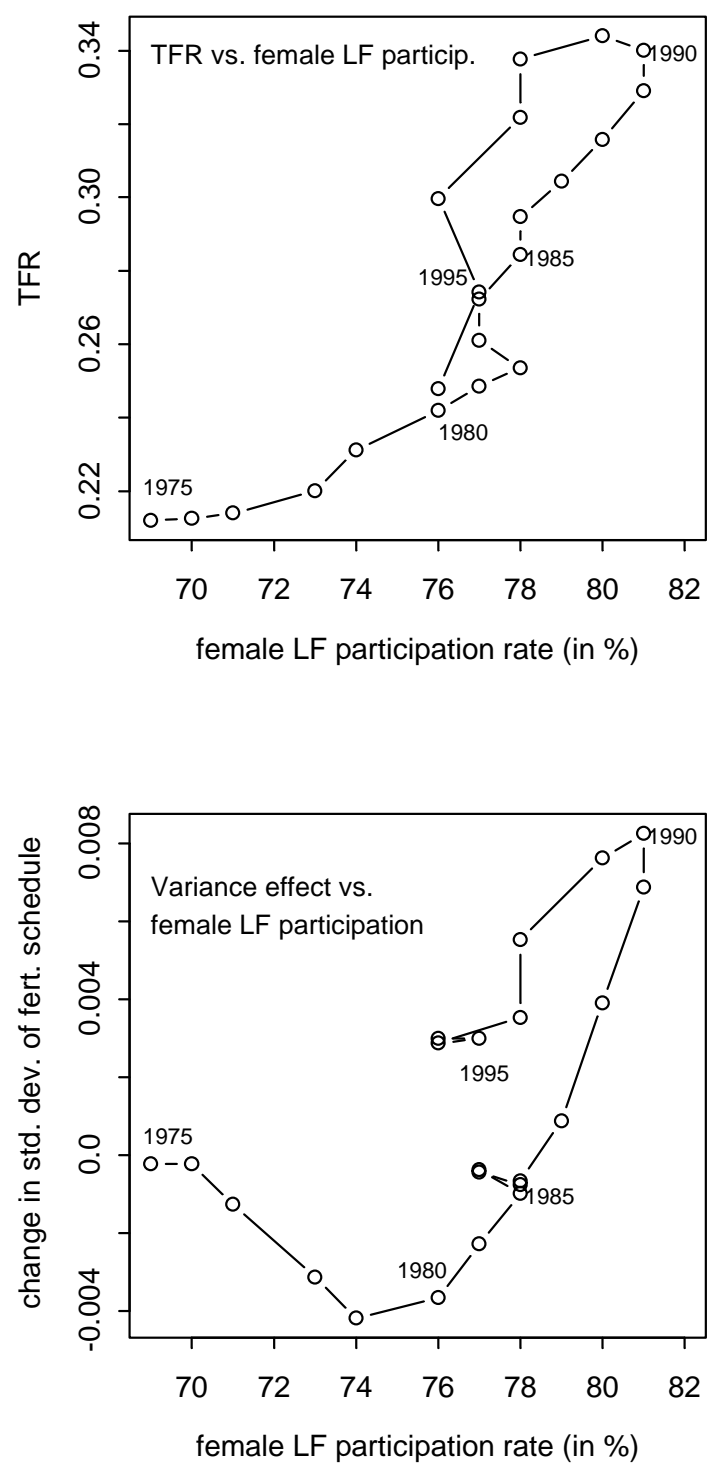

Figure 5: Female labor force participation and fertility patterns for first and third births in Sweden 
months of the previous child, the right to receive compensation for foregone income during childrearing was prolonged. Thus, parents had a short-term economic incentive to space births closely together. ${ }^{4}$ Hoem (1990) and Hoem and Hoem (1997b) argue that these policy changes are causally related to the increase in fertility rates relatively shortly after the birth of a previous child.

Because the analyses of policy effects by Hoem (1990) and Hoem and Hoem (1997b) are based on fertility rates by the age of the previous child, i.e., data that are not commonly available for countries other than Sweden, one should ask whether these policy effects can be identified with order-specific mean ages and the tempo effects derived from these mean ages. These latter data have the advantage of being available for many more countries, and they are used for the adjustment of the total fertility rate.

We will argue below that the differential implications of the policy changes for the timing of births are indeed detectable in the order-specific tempo effects - only, however, after the distortions caused by variance effects are removed. Consider, for instance, Figure 5. The bottom graphs juxtapose female labor force participation and the annual change in the standard deviation of the fertility schedule for first and third births. The latter reflects the extent of variance effects in the fertility pattern. For first births one can state that the increase in the female $L F P$ rate is not associated with a variance effect as long as the $T F R_{1}$ declines, i.e., until about 1984 . However, the joint increase in fertility and female $L F P$ after 1985 is associated with an increasing variance in the fertility schedule. Moreover, the extent of these variance effects increases over time: in 1987 the standard deviation of the fertility schedule increased by about $0.4 \%$ p.a., while in 1990 the annual change attained a maximum of $1.3 \%$.

This pattern suggests that the increase in fertility at an already high and increasing level of female labor force participation occurred via a diversification of fertility behavior. The fertility increase after 1984 was associated with a trend towards a wider dispersion of births across ages. The fertility increase was not due to a proportional increase in age-specific fertility rates at all ages, which would have left the variance of the fertility schedule unchanged. Instead, it was due to an increase in fertility rates that was more pronounced at ages above and below the mean age at first birth, thus leading to an increased variance of the fertility schedule (see also Hoem and Hoem 1997a for the differential increases in fertility by age and parity). This trend towards a wider dispersion of fertility across age was supported by various policy measures and public institutions that facilitated the combination of female labor force participation and fertility.

With the decline of the fertility rate for first births and the reduction in female labor force participation after 1990, the variance effects also diminish. The annual increase in the standard deviation of the fertility schedule dropped below $0.8 \%$ in 1995 (- 40\% compared to 1990). The tendency towards a higher standard deviation therefore remained even during the 1990s, despite the decline in fertility. However, the pace of this increase peaked in 1990 along with the fertility level 
$\left(T F R_{1}\right)$ and female labor force participation. Nevertheless, the lower fertility levels during the 1990s remain associated with moderate variance effects.

The pattern for third births is rather similar. The trend towards a reduced variance in the fertility schedule reversed in 1979, and after 1980 the increases in both $T F R_{3}$ and the female $L F P$ rate are associated with variance effects that tend to widen the fertility schedule for third births. As is the case for first births, the extent of these variance effects reaches a peak in 1990 along with the fertility level and female labor force participation. The baby bust since 1990 is associated with a slower pace (but not a reversal) of the trend towards an increased variance in the fertility schedule for third births.

In summary, Figure 5 suggests that there is a close connection between the increase in fertility during the late 1980s and the high and increasing levels of female labor force activity in Sweden. Public policies that favored the combination of work and children, along with a generally increased incentive for children during the late 1980s, seem to have caused a trend towards a diversification of fertility across ages.

These variance effects are interesting not only as a systematic component of the recent fertility pattern. They also facilitate the evaluation of the policy changes. In particular, accounting for these variance effects provides a more consistent picture of the parity-specific effects of the Swedish policy changes because the analyses clearly reflect the order-specific tempo changes associated with the extensions of the 'speed-premiums' introduced in 1980 and 1986.

Consider first the graph on the left in Figure 6, which depicts the tempo effects for first, second, and third births from 1975 to 1995 obtained from the application of the Bongaarts-Feeney adjustment. In this graph the tempo effect for first births is about 0.15 in the late 1970s with a minor increase around 1980. After 1983, the tempo effect for first births decreases substantially, leading to the plateau in the observed mean age in Figure 1. In 1988 the tempo effect for first births attains a minimum, then increases quite rapidly to a peak in 1992, followed by a renewed decline afterwards. The tempo for second births increases prior to 1989, and it then declines until 1989. The tempo for third births declines throughout the period 1975-1990.

The most striking aspect of the left graph in Figure 6 is that the tempo for all births decreases substantially in the years after 1983 without any significant differences in the pattern of tempo changes by birth order. One should have expected there to be differences, however, since the policy changes in 1980 and 1986 primarily affected the incentives for the timing of higher-order births.

The pattern of order-specific tempo effects changes quite substantially after the distortions caused by variance effects are removed. The right graph in Figure 6 reports the order-specific tempo effects after the changes in the shape of the fertility schedule have been accounted for.

The line for first births confirms our earlier finding that, after adjusting for variance effects, there was no substantial change in the tempo of fertility from 1975 to 1995 for first births. During 


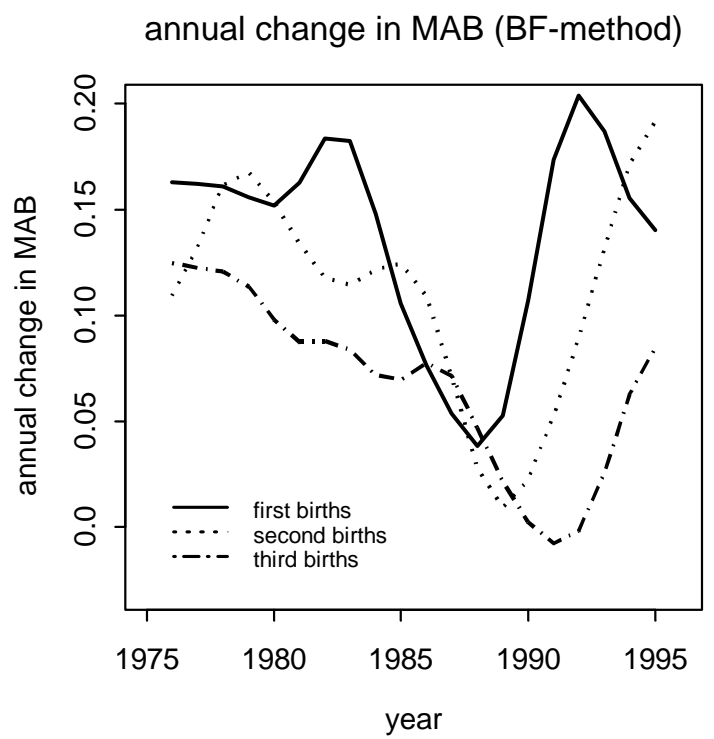

annual change in MAB w. variance effect

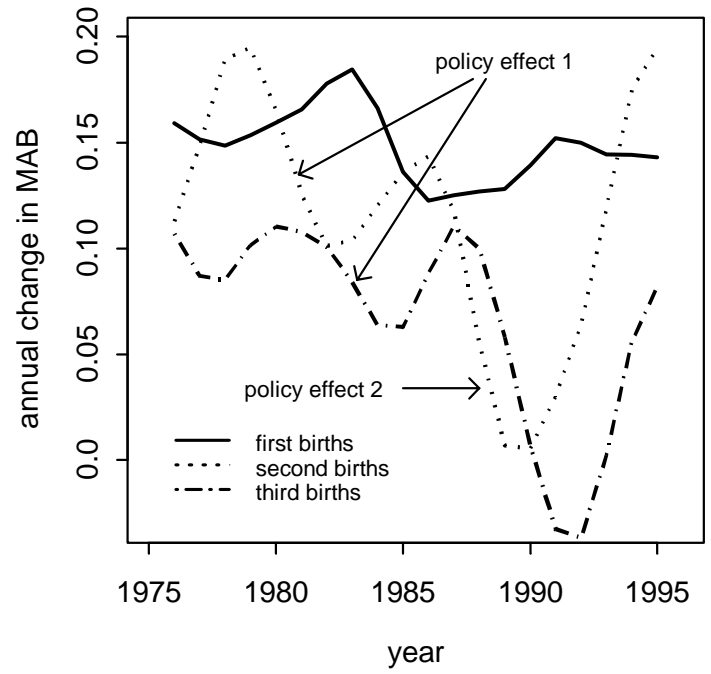

Figure 6: Annual change in the mean age at first, second, and third birth in Sweden, 1975-1995, based on the Bongaarts-Feeney adjustment of the TFR (left) and on the TFR adjustment with variance effects (right)

the period 1978-1985 the tempo first increases modestly and then decreases to about 0.12. Quite striking is the fact that during the period of the baby boom and bust (1985-1995) the tempo does not exhibit any substantial fluctuations. Throughout this period the mean age at first birth, adjusted for variance effects, increased by about $0.13-0.14$ years per annum.

For second and third births, however, substantial variations in tempo occur during the 1980s. After 1979, the upward trend that was present in the tempo for second births is reversed. The annual increases in the mean age at second birth decline by about 48\% from 1979 to 1982 (indicated by 'policy effect 1'). The slight increase after 1982 is probably an echo-effect of the increase in the tempo at first birth a few years earlier. After 1986, the tempo declines again substantially and almost reaches zero in 1990 (indicated by 'policy effect 2'). After 1990 the tempo for second births increases quite swiftly to a level prevailing in the late 1970s. The tempo for third births starts to decline modestly after 1980, followed by an increase which we believe is also due to an echo effect. After 1987 the tempo of third births decreases rapidly to a level slightly below zero and then increases after 1992.

If we account for the distortions caused by variance effects, this substantially alters the pattern of the tempo at different birth-orders during the 1980s. More importantly, the pattern after controlling for variance effects in the right graph in Figure 6 is more consistent with the policy changes during this period. The fluctuations in the tempo at order one are relatively small, which is consistent 
with the fact that the 'speed premium' has no direct implication for the timing of the first child. The policy changes do, however, provide incentives for shortening the interbirth interval after the second or third child, and the right graph in Figure 6 reflects clear decreases in the tempo for second or third births after 1980 and 1986. Since the tempo of first births (after accounting for variance effects) remains almost constant, this implies a reduction in the difference between the mean age at first and second birth, and between the mean age at first birth and third birth - both effects are consistent with, and are even an expected consequence of, the policy changes during the 1980s.

After variance effects are controlled for, the differential effect of the policy interventions in 1985 on births of different orders becomes clearly visible. Although the analyses in this paper do not contain information on interbirth intervals, the pattern obtained above is consistent with a reduction in the interbirth interval, especially between the first and second child. This pattern was detected earlier using different data, but it was not detectable from the trends in the observed mean ages without adjusting for variance effects.

\section{Concluding Remarks}

The adjusted total fertility rate suggested by Bongaarts and Feeney (1998) provides an additional and very useful measure for the analysis of fertility patterns, especially when fertility is subject to strong or fluctuating tempo effects. The adjusted total fertility rate is equivalent to the total fertility rate that would have been observed if there had been no postponement of births during a calender year. Since the postponement of births has been a characteristic of fertility declines in Western and Eastern Europe alike, the adjusted TFR exceeds - often substantially - the observed total fertility rate in countries with very low fertility levels (e.g., Bongaarts 1999; Philipov and Kohler 1999; Kohler and Philipov 1999). This finding is frequently associated with a cautious optimism that the completed fertility of women will exceed the level suggested by the observed $T F R$ and that fertility levels will rise once the postponement of births looses its momentum. ${ }^{5}$

Quite naturally, the adjustment of the total fertility rate needs to rely on assumptions in order to disentangle quantum and tempo components involved in changes in the total fertility rate. Probably the most severe assumption underlying the $T F R$-adjustment is that there are no age-period interactions or cohort effects. In other words, changes in the tempo of fertility are identical for all ages in any given period, and women of different ages cannot exhibit a different pace of postponement of their fertility.

Variance effects, i.e., increases in the standard deviation of the fertility schedule over time, result from the fact that in some recent European fertility patterns the tempo of fertility has differed for women of different ages. These variance effects therefore constitute a deviation from the assumptions underlying the TFR-adjustment. Moreover, Kohler and Philipov (1999) have shown that the presence of such variance effects leads to a bias in the mean age and in the adjusted total fertility 
rate. In order to make the $T F R$-adjustment applicable to countries with a changing variance of the fertility schedule, Kohler and Philipov (1999) provide an extension to the adjustment of the TFR that includes variance effects.

In this paper we apply the adjustment of the total fertility rate with and without variance effects to Sweden for the period 1975-1996. The Swedish fertility pattern during the 1980s and 1990s is most pertinent to the adjustment of the total fertility rate because the remarkable baby boom and bust during this period was associated with substantial changes in the tempo of fertility - at least when the tempo of fertility is inferred from changes in the observed (order-specific) mean age. We therefore address the question of the extent to which the baby boom and bust are due to tempo effects, i.e., changes in the postponement of fertility. We also question whether the mean age at birth reached a plateau during the late 1980s and whether the increase in fertility in the late 1980s was due to a reduction in the tempo of fertility. If this is the case, the Swedish fertility increase is exemplary for the type of fertility increases that are to be expected if the postponement of fertility comes to a halt in other countries.

The application of the Bongaarts and Feeney (1998) adjusted $T F R$ to Sweden suggests that in the years 1984-1988 the observed $T F R$ converges towards the adjusted $T F R$, which remains relatively constant during this period. This pattern indicates that the initial increase in the $T F R$ is due to a reduction in the tempo of fertility and hence does not represent an increase in the quantum of fertility. After 1988 the adjusted TFR initially rises - pointing towards an increase in the quantum of fertility - and then drops along with the observed $T F R$, which means that the recent decline in fertility is also due to quantum changes.

In this paper we show that the onset of the fertility increase around 1985 was associated with a tendency to spread childbirth more diversely across age: as fertility started to rise, the variance in the fertility schedule started to increase as well. This was most pronounced for first births, but was also evident to a somewhat more moderate extent for second and third births. We argue that these variance effects are related to a high - and even increasing - female labor force participation during the 1980s and to the social policy that facilitated the combination of labor force participation and childrearing.

In this situation, the upsurge in fertility initiated a diversification of the fertility behavior: the age-specific fertility rates did not rise proportionally across all ages, but they increased overproportionally at ages above and below the mean age at birth. This development manifested itself in an increased width of the fertility schedule. For instance, the standard deviation in the fertility schedule for first births increased by $9.7 \%$ (from 4.5 to above 4.9 ) from 1985 to 1995 , while the mean age at first birth increased by only $4.6 \%$ (from 26.1 to 27.3 years) in the same period.

The presence of these variance changes has substantial implications for the interpretation of the Swedish baby boom and bust in terms of tempo and quantum effects. After accounting for the 
distortions caused by variance changes, the plateau in the mean age at first birth in the late 1980s vanishes and the earlier association of the baby boom with a reduced pace in the postponement of fertility disappears. Instead, the tempo of fertility for first births remains almost constant throughout the period 1985-1995, indicating that neither the boom nor the bust in first births in Sweden was due to substantial changes in the tempo of fertility. After accounting for variance effects, the rise and decline in fertility for first births during this period is due almost entirely to quantum changes.

For second and third births the effects of variance changes are less marked. When all births are considered, however, the conclusion expressed above remains valid. Whereas the application of the Bongaarts and Feeney (1998) formula suggests that the Swedish baby boom is in part due to a reduced postponement of births, the adjusted $T F R$ with variance effects suggests that the baby boom and bust are predominantly due to quantum and not tempo changes.

Accounting for variance effects also reveals the differential impact of policy changes introduced during the 1980s on the timing of different birth orders. The major policy change of 1980 and 1986 is the extension of a so-called speed premium that benefited women/couples who had their next child within 24 (later 30) months of the previous child. This policy change directly altered the incentives for the timing of the second or third child, while it did not have a direct effect on the timing of the first child. While micro-data and fertility rates by order and age of first (second) child indicate that the policy changes in the 1980s caused a reduction in the interbirth interval between first and second, or second and third child, a corresponding pattern is not visible in the tempo calculated from mean ages at birth. The pattern of changes in the mean age does not differ significantly from birth order to birth order as one would expect, given the policy changes.

Once variance effects are included, this finding changes: the tempo effect for first births remains relatively constant during the period 1980-1995, while the tempo of second and third births declines substantially from 1985 to 1990 . Thus, the mean age at second or third birth increases at a considerably slower pace than the mean age at first birth, and consequently the difference between the mean age at first and second birth, is reduced. This means that after variance effects are accounted for, the pattern of tempo effects at different birth orders is consistent with the incentive changes caused by the policy changes: the tempo of fertility at first birth remains unaffected by the speed premium, while the tempo at higher orders is reduced.

In this paper we suggest that variance effects are a relevant aspect of fertility change in addition to tempo and quantum effects. While we fully agree that the adjustment of the total fertility rates provides new insights, the inferences are potentially distorted if the fertility change is associated with age-period interactions that change the shape of the fertility schedule over time. Correcting for changes in the variance makes it possible to incorporate changes in the fertility schedule in the adjustment of the total fertility rate. The Swedish example shows that inferences from the adjusted total fertility rate can differ substantially, depending on whether or not variance effects have been 
included in the analysis.

The Swedish fertility pattern is not unique with respect to the presence of variance effects. Increases in the variance of the fertility schedule have been associated with the fertility decline in Eastern Europe. Such increases have also occurred in Italy since the mid-1980s (Kohler and Philipov 1999; Philipov and Kohler 1999). The analysis of these patterns is beyond the scope of the present paper. Nevertheless we suggest that the changes in the variance of the fertility schedule can be a systematic, interesting and relevant aspect of fertility dynamics - one that deserves further consideration in the analysis of data from other countries as well.

\section{Notes}

${ }^{1}$ The data for the following analyses were obtained from the Eurostat (1998) NewCronos database. The age-specific fertility rates are calculated from the number of births by order and age of mother and the mid-year population in the respective years. The series for the $T F R$, the mean age, and the standard deviation of the fertility schedule were smoothed in all cases (using the robust running median smoother known as $4(3 \mathrm{RSR}) 2 \mathrm{H})$ before calculations were carried out, in order to remove minor and apparently random fluctuations from the respective series.

${ }^{2}$ The adjusted TFR in 1991-1992 actually exceeds the level of 1.0. This finding is not inconsistent with the fact that within a cohort each woman can have at most one first child. The adjusted TFR is a pure period measure, similar to the usual TFR. It is therefore not restricted by the contraceptive experience of cohorts, at least in the short run. It is thus perfectly consistent with the theory underlying the adjustment of the total fertility rate that the adjusted $T F R$ for first births exceeds one for a limited time period.

${ }^{3}$ The subsequent results are obtained by applying Results 9(a) and 10 in Kohler and Philipov (1999) to the smoothed order-specific series of the $T F R$, the mean age at birth, and the standard deviation of the fertility schedules for Sweden 1975-1996.

${ }^{4}$ The possibility of extending the eligibility interval via a close spacing of births also existed before 1980 , but the relatively short inter-birth interval of under 12 (or with sick leave 15) months that was required was frequently not attainable.

${ }^{5}$ It is important to note that the adjusted $T F R$, with or without correcting for variance effects, is a pure period measure which does not have direct implications for cohort fertility. See for instance the exchange between Kim and Schoen (1999) and Feeney and Bongaarts (1999). 


\section{References}

Andersson, G. (1999). Childbearing trends in Sweden 1961-1995. European Journal of Population 15(1), 1-24.

Bongaarts, J. (1999). Fertility decline in the developed world: Where will it end? American Economic Review 89(2), 256-260.

Bongaarts, J. and G. Feeney (1998). On the quantum and tempo of fertility. Population and Development Review 24(2), 271-291.

Council of Europe (1998). Recent Demographic Developments in Europe. Strasbourg: Council of Europe Publishing.

Eurostat (1998). New Cronos Database - Population and Social Conditions. Brussels: Eurostat.

Feeney, G. and J. Bongaarts (1999). Reply to Kim and Schoen. Unpublished manuscript (available at http://gfeeney.com).

Gustafsson, S. S., C. M. M. P. Wetzels, J. D. Vlasblom, and S. Dex (1996). Women's labor force transitions in connection with childbirth: a panel data comparison between Germany, Sweden and Great Britain. Journal of Population Economics 9(3), 223-246.

Hoem, B. (1998). Entry into motherhood in Sweden: The influence of economic factors on the rise and fall in fertility, 1986-1997. Paper presented at the workshop on Lowest Low Fertility, Max Planck Institute for Demographic Research, Rostock, Germany, December 10-11, 1998.

Hoem, B. and J. M. Hoem (1997a). Fertility trends in Sweden up to 1996. Population Bulletin (United Nations), forthcoming.

Hoem, B. and J. M. Hoem (1997b). Sweden's family policies and roller-coaster fertility. Journal of Population Problems 52(3-4), 1-22.

Hoem, J. M. (1990). Social policy and recent fertility change in Sweden. Population and Development Review 16(4), 735-48.

Kim, Y. J. and R. Schoen (1999). Changes in timing and the measurement of fertility. Paper presented at the Annual Meeting of the Population Association of America, New York, March $24-27$.

Kohler, H.-P. and D. Philipov (1999). Variance effects and nonlinearities in the Bongaarts-Feeney formula. Unpublished manuscript. An earlier version of the paper is available as Max Planck Institute for Demographic Research Working Paper \#1999-001.

Meisaari-Polsa, T. and L. Söderström (1995). Recent Swedish fertility changes in perspective. In C. Lundh (Ed.), Demography, Economy and Welfare, pp. 11-27. Lund, Sweden: Lund University Press. 
OECD (1998). Labor Market Database. Paris, France: Organisation for Economic Co-operation and Development.

Philipov, D. and H.-P. Kohler (1999). The relevance of tempo effects for the fertility decline in Eastern Europe: Evidence from Bulgaria, the Czech Republic, Hungary, Poland and Russia. Paper presented at the workshop on Lowest Low Fertility at the Max Planck Institute for Demographic Research, Rostock, Germany, December 1998.

Sundstrom, M. and F. P. Stafford (1992). Female labour force participation, fertility and public policy in Sweden. European Journal of Population 8(3), 199-215.

van de Kaa, D. J. (1987). Europe's second demographic transition. Population Bulletin 42(1), $1-57$.

Walker, J. R. (1995). The effect of public policies on recent Swedish fertility behavior. Journal of Population Economics 8(3), 223-51. 